\title{
Bridgman Growth and Photoelectronic Property of Relaxor-Based Ferroelectric Single Crystal $\mathrm{Pb}\left(\mathrm{Sm}_{1 / 2} \mathrm{Nb}_{1 / 2}\right) \mathrm{O}_{3}-\mathrm{Pb}\left(\mathrm{Mg}_{1 / 3} \mathrm{Nb}_{2 / 3}\right) \mathrm{O}_{3}-\mathrm{PbTiO}_{3}$
}

\author{
Fan Liao ${ }^{1}$, Yan Zhao ${ }^{1}$, Ziyun Chen ${ }^{2}$, Yanqing Zheng ${ }^{1, *}$ and Hongbing Chen ${ }^{1, *}$ \\ 1 State Key Base of Functional Material \& Its Preparation Science, Key Laboratory of Photoelectric Detection \\ Materials and Devices of Zhejiang Province, Institute of Materials Science \& Chemical Engineering, \\ Ningbo University, Ningbo 315211, China; frank_leon@163.com (F.L.); zhaoyan983@163.com (Y.Z.) \\ 2 Department of Instrument Science and Engineering, Shanghai Jiao Tong University, Shanghai 200240, China; \\ chenzy_james@sjtu.edu.cn \\ * Correspondence: zhengyanqing@nbu.edu.cn (Y.Z.); chenhongbing@nbu.edu.cn (H.C.)
}

check for updates

Citation: Liao, F.; Zhao, Y.; Chen, Z.; Zheng, Y.; Chen, H. Bridgman Growth and Photoelectronic Property of Relaxor-Based Ferroelectric Single Crystal $\mathrm{Pb}\left(\mathrm{Sm}_{1 / 2} \mathrm{Nb}_{1 / 2}\right) \mathrm{O}_{3}$ $\mathrm{Pb}\left(\mathrm{Mg}_{1 / 3} \mathrm{Nb}_{2 / 3}\right) \mathrm{O}_{3}-\mathrm{PbTiO}_{3}$. Crystals 2021, 11, 402. https://doi.org/ $10.3390 /$ cryst11040402

Academic Editors: Shujun Zhang, Guisheng $\mathrm{Xu}$ and Yizheng Tang

Received: 12 March 2021

Accepted: 5 April 2021

Published: 10 April 2021

Publisher's Note: MDPI stays neutral with regard to jurisdictional claims in published maps and institutional affiliations.

Copyright: (c) 2021 by the authors. Licensee MDPI, Basel, Switzerland. This article is an open access article distributed under the terms and conditions of the Creative Commons Attribution (CC BY) license (https:// creativecommons.org/licenses/by/ $4.0 /)$.
Abstract: A relaxor-based ferroelectric single crystal with the nominal composition of $x \mathrm{~Pb}\left(\mathrm{Sm}_{0.5} \mathrm{Nb}_{0.5}\right) \mathrm{O}_{3}$ (0.7-x) $\mathrm{Pb}\left(\mathrm{Mg}_{1 / 3} \mathrm{Nb}_{1 / 3}\right) \mathrm{O}_{3}-0.3 \mathrm{PbTiO}_{3}(\mathrm{x}=0.01,0.02$, and 0.03$)$ was grown by the vertical Bridgman process. The electrical properties and the ferroelectric domains, as well as the luminescent characteristics of the single crystals, were investigated systematically. The piezoelectric coefficient $d_{33}$ of the single crystals are slightly higher than that of the undoped PMN-PT single crystal under direct current polarization, while the crystal wafers gain a much higher $d_{33}$ value upon being polarized with alternating current voltage. The single crystals possess a decreased phase transition temperature of around $60{ }^{\circ} \mathrm{C}$ and a decreased Curie temperature of $92 \sim 116^{\circ} \mathrm{C}$ compared with the undoped PMN-PT single crystal. The crystal wafers polarized with alternating current voltage exhibited a desirable optical transmittance, which is associated with the domain structure changes inside the crystal medium. The domain density of the crystal wafers under alternating current polarization was significantly decreased compared with the direct current polarized crystal wafers. The luminescent spectra of the crystal wafers exhibit the typical emission peaks corresponding to the characteristic transition of $\mathrm{Sm}^{3+}$ ions in the crystal lattice.

Keywords: relaxor-based ferroelectric single crystal; crystal growth; electrical property; luminescent properties

\section{Introduction}

For a long time, traditional lead-based piezoelectric materials, such as $\mathrm{Pb}(\mathrm{Zr}, \mathrm{Ti}) \mathrm{O}_{3}$ (PZT) ceramics, were widely used in piezoelectric devices due to their better piezoelectric properties, good temperature stability, and mature manufacturing processes. For example, the piezoelectric coefficient and Curie temperature of $\mathrm{PZT} 5 \mathrm{H}$ piezoelectric ceramic are $600 \mathrm{pC} / \mathrm{N}$ and $200{ }^{\circ} \mathrm{C}$, respectively [1-3]. In the past decade, the relaxor-based ferroelectric single crystals, such as $\mathrm{Pb}\left(\mathrm{Mg}_{1 / 3} \mathrm{Nb}_{2 / 3}\right) \mathrm{O}_{3}-\mathrm{PbTiO}_{3}(\mathrm{PMN}-\mathrm{PT})$ and $\mathrm{Pb}\left(\mathrm{Zn}_{1 / 3} \mathrm{Nb}_{2 / 3}\right) \mathrm{O}_{3}-$ $\mathrm{PbTiO}_{3}$ (PZN-PT), have been investigated as novel piezoelectric materials with ultrahigh piezoelectric performance [4-6]. The relaxor-based ferroelectric single crystals have been proven to be especially valuable for the applications of piezoelectric devices, such as medical ultrasonic imaging probes, ultrasonic actuators, and sonar transducers [7-10]. In the past decade, more systematic research work has been performed around the $\mathrm{PbTiO}_{3}$-based material to acquire even better electrical properties, especially giant piezoelectricity, to meet the technical demands for highly sensitive transducers.

Recently, many studies have focused on the rare earth ions-modified relaxor ferroelectric crystals due to their excellent electrical properties [11-17]. For instance, the single crystal $0.31 \mathrm{~Pb}\left(\mathrm{Mg}_{1 / 3} \mathrm{Nb}_{2 / 3}\right) \mathrm{O}_{3}-0.34 \mathrm{~Pb}\left(\mathrm{Lu}_{1 / 2} \mathrm{Nb}_{1 / 2}\right)-0.35 \mathrm{PbTiO}_{3}$ grown by Liu et al. has 
outstanding electric properties, including the piezoelectric coefficient of $2092 \mathrm{pC} / \mathrm{N}$ and a Curie temperature of $228^{\circ} \mathrm{C}$ [13]. Another new $\mathrm{Pb}\left(\mathrm{Mg}_{1 / 3} \mathrm{Nb}_{2 / 3}\right) \mathrm{O}_{3}-\mathrm{Pb}\left(\mathrm{Yb}_{1 / 2} \mathrm{Nb}_{1 / 2}\right)-\mathrm{PbTiO}_{3}$ crystal was grown by the top-seeded solution growth method [14], with the piezoelectric coefficient and the Curie temperature reaching $1800 \mathrm{pC} / \mathrm{N}$ and $205^{\circ} \mathrm{C}$, respectively. Recently, Li et al. prepared $\mathrm{Sm}$-doped $\mathrm{Pb}\left(\mathrm{Mg}_{1 / 3} \mathrm{Nb}_{2 / 3}\right) \mathrm{O}_{3}-\mathrm{PbTiO}_{3}(\mathrm{PMN}-\mathrm{PT}$ ) perovskite ferroelectric ceramics [17]. The ultrahigh piezoelectric coefficients $d_{33}$ was up to $1500 \mathrm{pC} / \mathrm{N}$ and dielectric permittivity $\varepsilon_{\mathrm{r}}$ was above 13,000 with a Curie temperature $\left(\mathrm{T}_{\mathrm{c}}\right)$ of $89{ }^{\circ} \mathrm{C}$. Lately, Li et al. proved that $\mathrm{Sm}^{3+}$-doped PMN-PT single crystal, in which $\mathrm{Sm}^{3+}$ ions were doped into the A sites inside the crystal lattice, possesses even higher piezoelectric coefficient $d_{33}$ values, ranging from 3400 to $4100 \mathrm{pC} / \mathrm{N}$ [18]. The giant piezoelectricity of the $\mathrm{Sm}^{3+}$-doped PMN-PT single crystal was attributed to the enhanced local structural heterogeneity introduced by A-sites-located $\mathrm{Sm}^{3+}$ dopants.

The relaxor-based ferroelectric materials, including $\mathrm{PbTiO}_{3}$-based solid solution ceramics and single crystals, have been continuously investigated in our laboratory over the past few years $[19,20]$. Our previous investigations were performed on the rare-earthdoped $\mathrm{PbTiO}_{3}$-based materials, such as $\mathrm{Er}^{3+}$-doped PMN-PT single crystal, grown by the vertical Bridgman process, which were measured to exhibit improved electrical properties as well as up-conversion luminescence properties. In this paper, we report a ternary solid solution single crystal with the nominal formula of $x \mathrm{PSN}-(0.70-\mathrm{x}) \mathrm{PMN}-0.30 \mathrm{PbTiO}_{3}$ grown by the vertical Bridgman process. By adding another component, $\mathrm{Pb}\left(\mathrm{Sm}_{1 / 2} \mathrm{Nb}_{1 / 2}\right) \mathrm{O}_{3}$ with appropriate ratios, $\mathrm{Sm}^{3+}$ ions were doped into the $\mathrm{B}$ sites in the ternary solid solution single crystal PSN-PMN-PT. The single crystals with different PSN component ratios were characterized systematically concerning dielectric, piezoelectric, and ferroelectric properties as well as the fluorescence emission.

\section{Experimental Procedure}

\subsection{Polycrystalline Material Synthesis}

According to the composition ratios of $x \mathrm{~Pb}\left(\mathrm{Sm}_{1 / 2} \mathrm{Nb}_{1 / 2}\right)-(0.7-\mathrm{x}) \mathrm{Pb}\left(\mathrm{Mg}_{1 / 3} \mathrm{Nb}_{2 / 3}\right) \mathrm{O}_{3}-$ $0.3 \mathrm{PbTiO}_{3}$, a series of polycrystalline materials with $\mathrm{x}=0.01,0.02$, and 0.03 was prepared by a solid-state reaction at an elevated temperature. The raw reagents of $\mathrm{Sm}_{2} \mathrm{O}_{3}, \mathrm{PbO}$, $\mathrm{Nb}_{2} \mathrm{O}_{5}, \mathrm{TiO}_{2}$, and $4 \mathrm{MgCO}_{3} \cdot \mathrm{Mg}(\mathrm{OH})_{2} \cdot 4 \mathrm{H}_{2} \mathrm{O}$ with $99.99 \%$ purity were used to prepare the polycrystalline materials via the two-step columbite precursor route. Firstly, the precursors $\mathrm{SmNbO}_{4}$ and $\mathrm{MgNb}_{2} \mathrm{O}_{6}$ were prepared by mixing $4 \mathrm{MgCO}_{3} \cdot \mathrm{Mg}(\mathrm{OH})_{2} \cdot 4 \mathrm{H}_{2} \mathrm{O}, \mathrm{Sm}_{2} \mathrm{O}_{3}$, and $\mathrm{Nb}_{2} \mathrm{O}_{5}$ at the stoichiometric molar ratio, and then sintered at $1100{ }^{\circ} \mathrm{C}$ for $6 \mathrm{~h}$. Subsequently, the oxide reagents of $\mathrm{TiO}_{2}$ and $\mathrm{PbO}(0.1$ mol \% excess) were mixed uniformly with the precursors $\mathrm{SmNbO}_{4}$ and $\mathrm{MgNb}_{2} \mathrm{O}_{6}$ in an alcohol medium, and then sintered at $850{ }^{\circ} \mathrm{C}$ for $6 \mathrm{~h}$. A polycrystalline powder with the composition of xPSN-(0.7-x) PMN-0.3PT $(x=0.01$, 0.02 , and 0.03 ) was obtained finally by the solid-state synthesis process above.

\subsection{Crystal Growth}

A series of PSN-PMN-PT single crystals with different ratios of PSN components were grown in a vertical Bridgman furnace with resistive heating elements. The specially fabricated platinum crucibles, $\varnothing 30 \times 220 \mathrm{~mm}$ in dimension, were used in the growing process. Using PMN-PT seed crystals with a size of $\varnothing 11.6 \times 50 \mathrm{~mm}$ installed in the seed wells, the oriented crystal growth with (110)-crystallographic direction was performed. After the polycrystalline materials were filled above the seed crystals, the charged crucibles were sealed to avoid the volatilization of melts during the crystal growth. The specially designed furnace with three temperature zones was controlled by a WJK-100A temperature meter, with an accuracy of $\pm 0.5^{\circ} \mathrm{C}$. After the charged crucibles installed in the refractory tubes were placed into the furnace chamber, the high temperature zone of the furnace chamber was raised to the controlled temperature of $1370-1380{ }^{\circ} \mathrm{C}$. The oriented crystal growth was firstly performed via the seeding process operated by adjusting the crucible to an appropriate position. The crystallization process was then driven forward along the crucible cylinder as the crucibles descended at a rate of $10 \mathrm{~mm} /$ day. As the growth 
procedure for more than ten days had been finished, the furnace was finally cooled down to room temperature at a rate of $40-60^{\circ} \mathrm{C} / \mathrm{h}$. As-grown crystal boules were obtained by stripping the platinum crucibles.

\subsection{Characterization}

The powder samples collected from the polycrystalline material and the single crystal were analyzed by an X-ray diffractometer (XRD, D8 Advance, Bruker, Billerica, MA, USA) with $\mathrm{Cu} \mathrm{K} \mathrm{K}_{\alpha}$ radiation. A series of crystal wafers, labeled (a), (b), and (c), were cut from the middle region of as-grown crystal boules. The (001)-oriented crystal wafers were polished to $0.8 \mathrm{~mm}$ thickness, and both sides were coated with a silver film of electrodes and then sintered at $600{ }^{\circ} \mathrm{C}$ for $6 \mathrm{~h}$. The crystal wafers were polarized by direct current (DC) or alternating current $(\mathrm{AC})$ voltage. For DC polarization, the samples were dwelt for $10 \mathrm{~min}$ at a field of $12 \mathrm{kV} / \mathrm{cm}$. For AC polarization, a symmetrical triangular wave AC voltage was applied to the samples, with a peak value of electric field intensity of $12 \mathrm{kV} / \mathrm{cm}$, a frequency of $10 \mathrm{~Hz}$, and a cycle number of 15, which were generated by a functional generator (Agilent 33220A) and amplified by a high-voltage amplifier (Trek Model 10/40A). All the samples were immersed in silicone oil during the poling process, at a pooling temperature of $30-50{ }^{\circ} \mathrm{C}$. The dielectric permittivity $\varepsilon$ and dielectric loss $\tan \delta$ were tested using an Agilent $4294 \mathrm{~A}$ impedance analyzer at various temperatures $\left(30-300^{\circ} \mathrm{C}\right)$ and at $100 \mathrm{~Hz}, 1 \mathrm{kHz}$, $10 \mathrm{kHz}$, and $100 \mathrm{kHz}$ frequencies. The piezoelectric constant $d_{33}$ of samples were measured by a quasi-static piezoelectric meter (ZJ-3AN, Institute of Acoustics, Chinese Academy of Science, Beijing, China). The polarization-electric field (P-E) hysteresis loops were measured using a ferroelectric analyzer (Premier II, Radiant Technologies, Albuquerque, NM, USA) under the frequency of $1 \mathrm{~Hz}$ at room temperature. The bright-field TEM and differential phase contrast (DPC) images observations were carried out using a Talos F200X G2 microscope operated at $200 \mathrm{kV}$. The transmittance spectra were measured via a spectrophotometer (PerkinElmer Lambda 950, Waltham, MA, USA), and the luminescence spectra were tested using a fluorescence spectrometer (FLS980, Edinburgh, England).

\section{Results and Discussion}

\subsection{Crystallographic Characterization of Crystal Boules}

Using the polycrystalline materials with the composition ratios of $x \mathrm{PSN}-(0.70-\mathrm{x}) \mathrm{PMN}-$ $0.30 \mathrm{PT}(\mathrm{x}=0.01,0.02,0.03)$, a series of single crystals with perovskite structure was grown successfully by the vertical Bridgman process. Figure 1 shows three crystal boules with dimensions of $\varnothing 30 \times 70 \mathrm{~mm}$ or so grown via the process described above. The cylindric crystal boules appeared to be translucent and light brown in color. Figure 2 shows the XRD patterns of polycrystalline powders and crystal powders, which are in good agreement with previous reports [21-23]. Although the polycrystalline material contained a little amount of pyrochlore phase, as a weak diffraction peak appeared at $20-29^{\circ}$, no diffraction peak of the secondary phase was found, indicating that the $\mathrm{Sm}^{3+}$ ions had been well-diffused into the lattices of crystals. It demonstrated the pure perovskite phase of the as-grown crystal without any undesired pyrochlore phase. Considering that $\mathrm{Sm}^{3+}$ ions are doped via the solid solution component $\mathrm{Pb}\left(\mathrm{Sm}_{1 / 2} \mathrm{Nb}_{1 / 2}\right), \mathrm{Sm}^{3+}$ ions were confirmed to be located in the $\mathrm{B}$ sites of the perovskite crystal. In the literature, $\mathrm{Li}$ et al. reported $\mathrm{Sm}^{3+}$-doped PMN-PT single crystal prepared by directly adding $\mathrm{Sm}_{2} \mathrm{O}_{3}$ to PMN-PT, in which $\mathrm{Sm}^{3+}$ ions are thought to be located in A sites. 


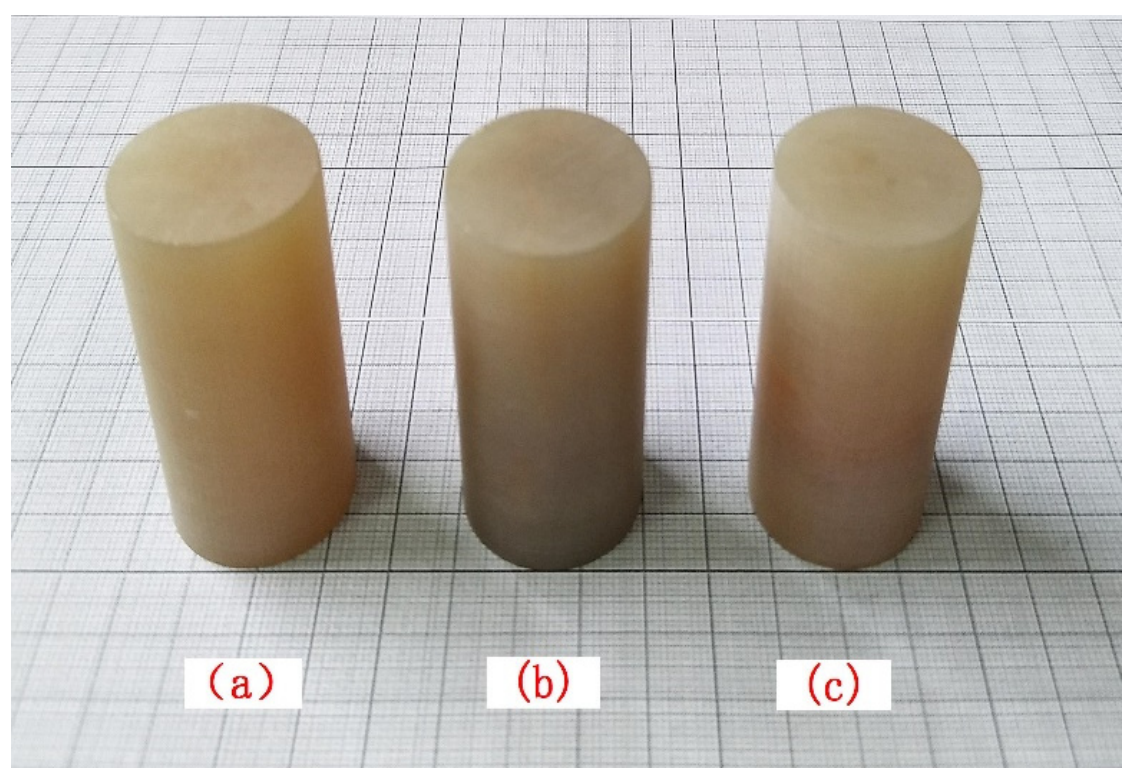

Figure 1. The crystal boules grown by the vertical Bridgman process from the polycrystalline materials with the composition ratios of (a) 0.01PSN-0.69PMN-0.30PT (b) 0.02PSN-0.68PMN-0.30PT and (c) 0.03PSN-0.67PMN-0.30PT.

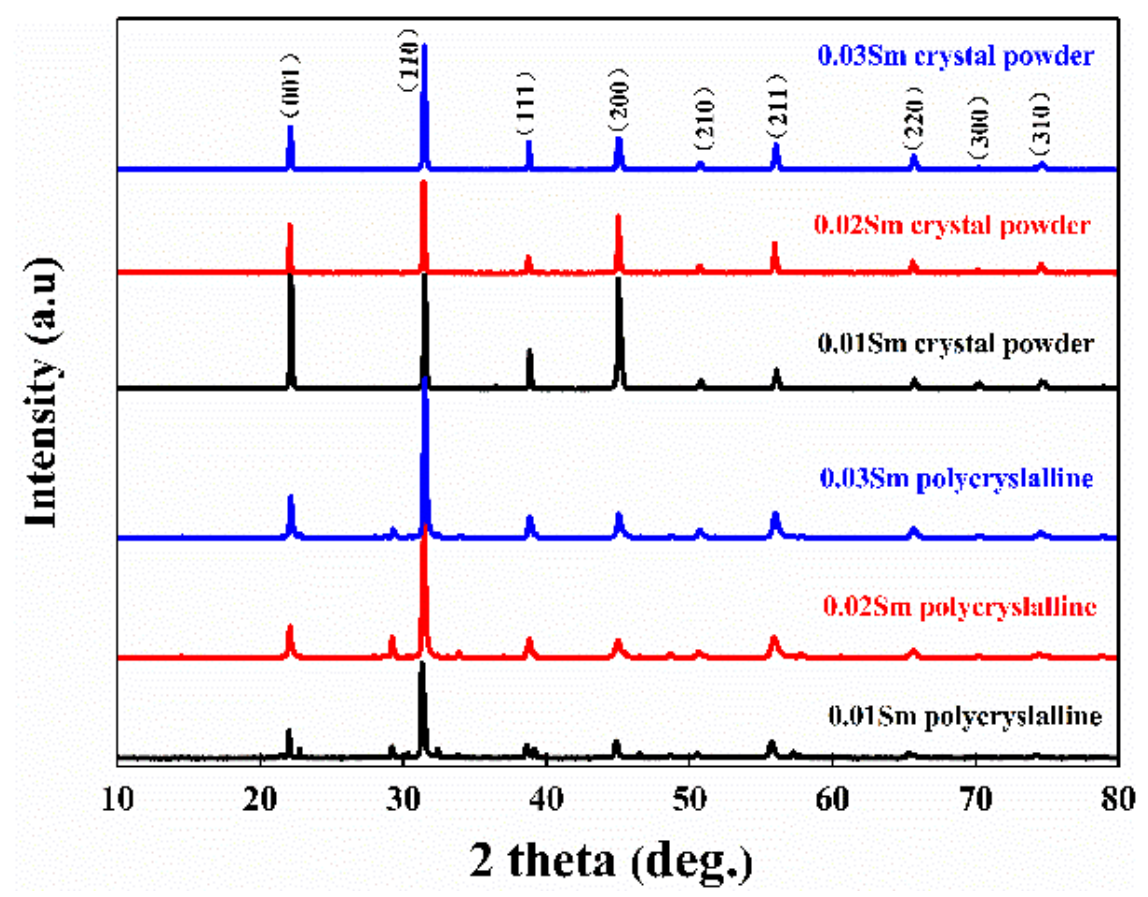

Figure 2. XRD patterns of the polycrystalline powder and the crystal powder.

\subsection{Dielectric Behavior}

Figure $3 \mathrm{a}-\mathrm{c}$ presents the temperature dependence of the dielectric permittivity of the crystal samples (a), (b), and (c) at different frequencies (100 Hz 100 KHz), respectively. The crystal wafers fabricated from the middle region of the crystal boules (a), (b), and (c) were measured to investigate their dielectric behavior. It can be seen that the anomalous dielectric peaks at the Curie temperature $\left(T_{c}\right)$ are relatively wide. As the frequency increased, the peaks moved toward the high temperatures, and the maximum dielectric constants gradually decreased, indicating the relaxation behaviors of present crystals. Figure $3 a$, corresponding to the crystal boule (a), presents three anomalous dielectric peaks, i.e., peak I at $59{ }^{\circ} \mathrm{C}$, peak II at $74{ }^{\circ} \mathrm{C}$, and peak III at $116{ }^{\circ} \mathrm{C}$. Peak I was attributed to the 
rhombohedral to orthorhombic phase transition, while peak II and peak III were associated with the phase transition of the orthorhombic to tetragonal and tetragonal-cubic phase, respectively [15]. Figure 3b,c, corresponding to the crystal boules (b) and (c) respectively, shows two anomalous dielectric peaks, corresponding to the rhombohedral to tetragonal and the tetragonal to cubic phase transitions, respectively. In contrast with pure PMN-PT single crystal, the single crystals with $\mathrm{Sm}^{3+}$ ions doped at B sites exhibit a lower phase temperature and a lower Curie temperature [24].
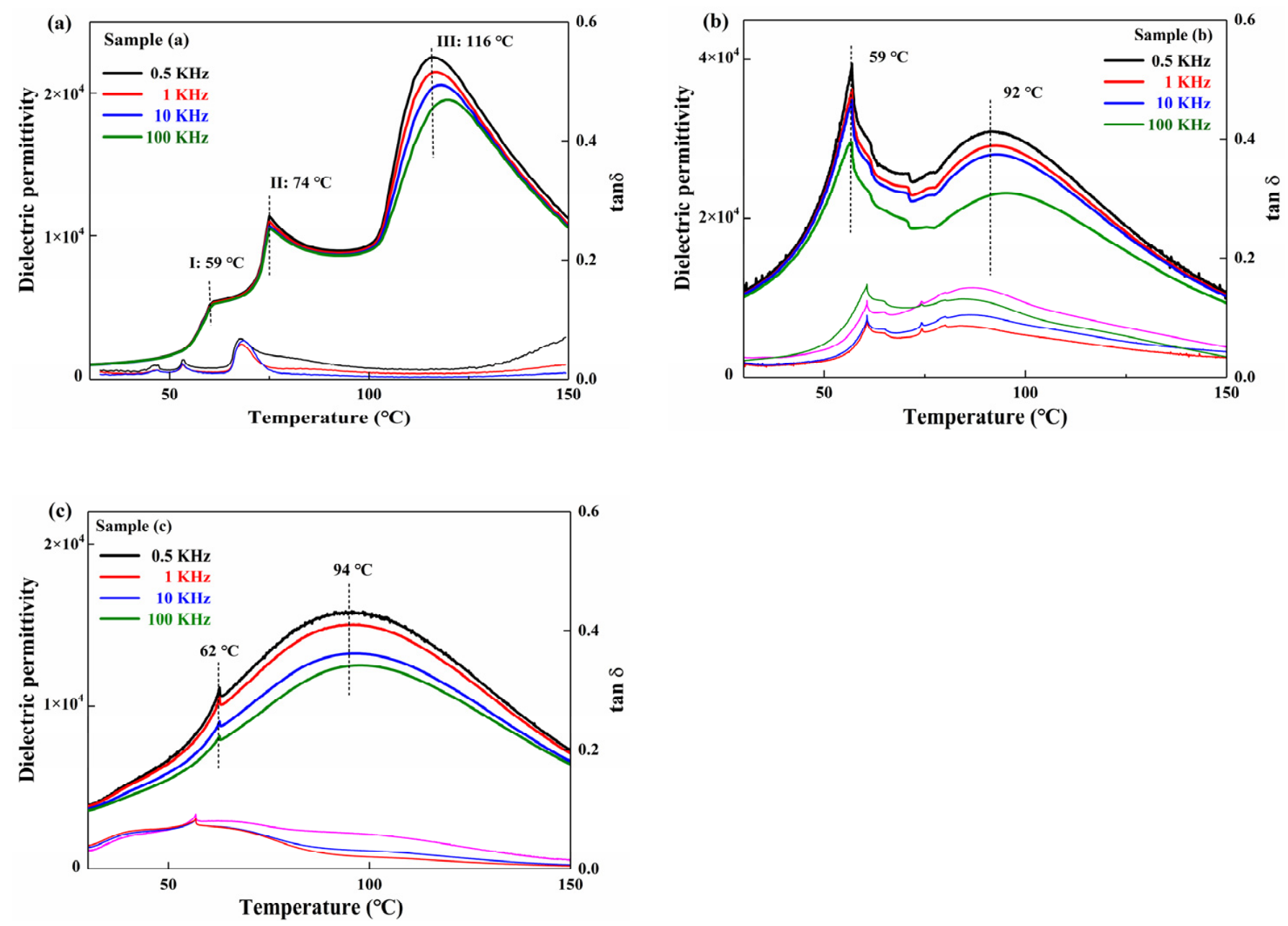

Figure 3. The temperature dependence of dielectric permittivity and dielectric loss tan $\delta$ for the crystal wafers fabricated from the different crystal samples (a), (b), and (c).

\subsection{Piezoelectric Coefficient and Ferroelectric Property}

The crystal wafers fabricated from the middle region of crystal boules (a), (b), and (c) were measured to investigate the piezoelectric property after the samples were subjected to the direct current $(\mathrm{DC})$ or the alternating current $(\mathrm{AC})$ polarization. The piezoelectric coefficients $d_{33}$ for these crystal wafers acquired in the measurement are illustrated with the listed data in Table 1. We verified that the single crystals under DC polarization in this work exhibited a piezoelectric coefficient $d_{33}$, somewhat higher in varying degrees than those for the undoped PMN-PT single crystal, with the average $d_{33}$ value being approximately in the range of $1100 \sim 1800 \mathrm{pC} / \mathrm{N}$ [25]. It is notable that the single crystals under AC polarization have evidently increased $d_{33}$ piezoelectric coefficients compared with those under DC polarization. Among the three single crystals, the crystal sample (b) possessed even higher piezoelectric coefficients compared with those for the crystal samples under either DC or AC polarization. The crystal sample with the nominal composition of $0.02 \mathrm{PSmN}-0.68 \mathrm{PMN}-$ 
0.30PT possessed a maximal piezoelectric coefficient as high as 3100-3600 pC/N after it was polarized by AC voltage.

Table 1. The piezoelectric coefficient $d_{33}$ of xPSN-(0.7-x)PMN-0.3PT single crystals.

\begin{tabular}{cccc}
\hline Sample No. & Nominal Composition & $\boldsymbol{d}_{\mathbf{3 3}}$ under DC Voltage (pC/N) & $\boldsymbol{d}_{\mathbf{3 3}}$ under AC Voltage (pC/N) \\
\hline (a) & $0.01 \mathrm{PSmN-0.69PMN-0.30PT}$ & $1730 \sim 2020$ & $2560 \sim 3100$ \\
(b) & $0.02 \mathrm{PSmN-0.68PMN-0.30PT}$ & $1960 \sim 2380$ & $3100 \sim 3600$ \\
(c) & $0.03 \mathrm{PSmN-0.67PMN-0.30PT}$ & $1780 \sim 2100$ & $2480 \sim 3300$ \\
\hline
\end{tabular}

Figure 4 displays the polarization versus electric field (P-E) hysteresis loops of the crystal samples (a), (b), and (c) under the frequency of $1 \mathrm{~Hz}$ at room temperature. A typical ferroelectric hysteresis loop was observed at an electric field of $1 \mathrm{kV}$. The crystal samples (a), (b), and (c) were measured to possess a coercive field $\left(\mathrm{E}_{\mathrm{c}}\right)$ of $3.61,1.44$, and $1.58 \mathrm{kV} / \mathrm{cm}$, and a remnant polarization of $14.7,22.31$, and $23.71 \mu \mathrm{C} / \mathrm{cm}^{2}$, respectively. Compared with the undoped PMN-PT single crystal, the ternary solid state solution single crystals exhibited a lower coercive field.

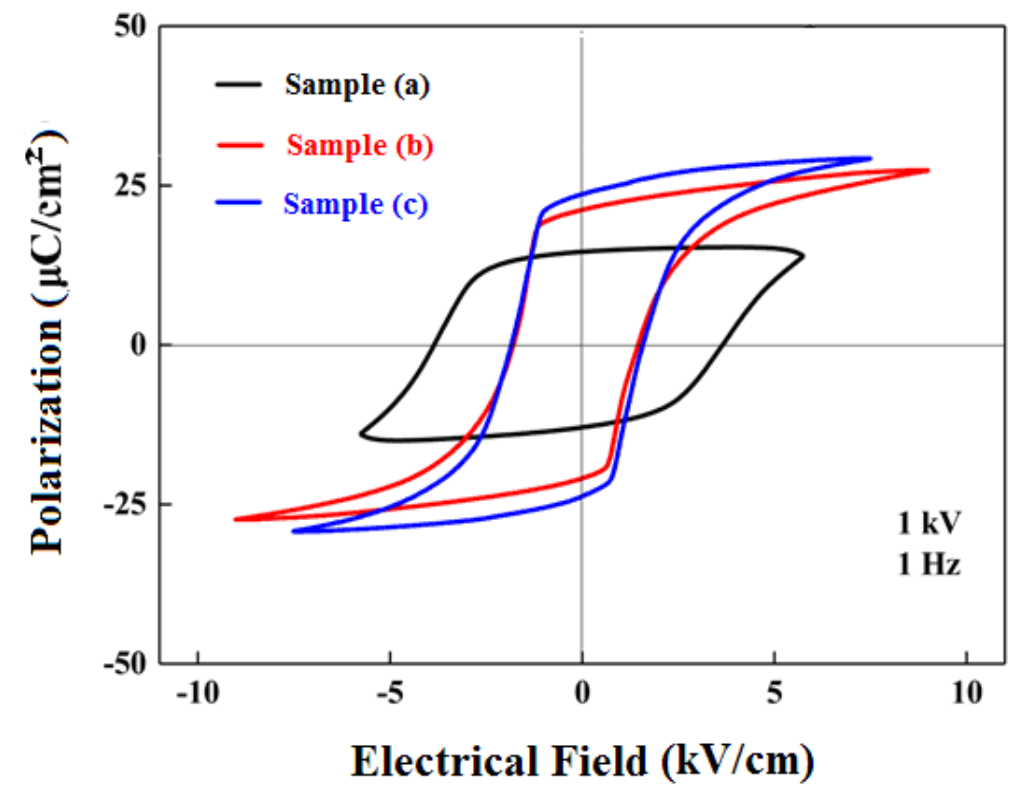

Figure 4. The polarization versus electric field $(\mathrm{P}-\mathrm{E})$ of the different crystal wafers.

\subsection{Domain Structure Observation}

The ferroelectric domain structure of the $0.02 \mathrm{PSmN}-0.68 \mathrm{PMN}-0.30 \mathrm{PT}$ single crystal is shown in Figure 5. It can be observed that the polarized samples have a band-like fringe domain wall structure. The domain density of crystals under direct current polarization (DCP) in Figure $5 c$,d is significantly higher than that under alternating current polarization $(\mathrm{ACP})$ in Figure $5 \mathrm{a}, \mathrm{b}$. The results suggest that the crystal samples subjected to ACP exhibited lower domain density and less domain types.

The deflection direction and the distribution of electric domains in the samples were measured by differential phase contrast (DPC). The results of DPC analysis on the crystals are shown in Figure 6, in which colors represent different directions of the electric field, and the arrows represent the directions of the electric fields. It is observed that the domain density decreases as the domain size is increases, as the crystal wafers were polarized with AC voltage. It can be explained by the alternating of the polarity of the electric field lowering the free energy of ferroelectric crystals, leading to the formation of a new domain structure with reduced domain wall density. The experimental results in our work are also consistent with the investigations by the recent literature. Li et al. [26] demonstrated by phase-field simulations that the application of an AC electric field reduced the number of 
$71^{\circ}$ domain walls effectively, and only two $71^{\circ}$ domain walls were left in each lamina after AC polarization, which led to a much larger domain size within each lamina.
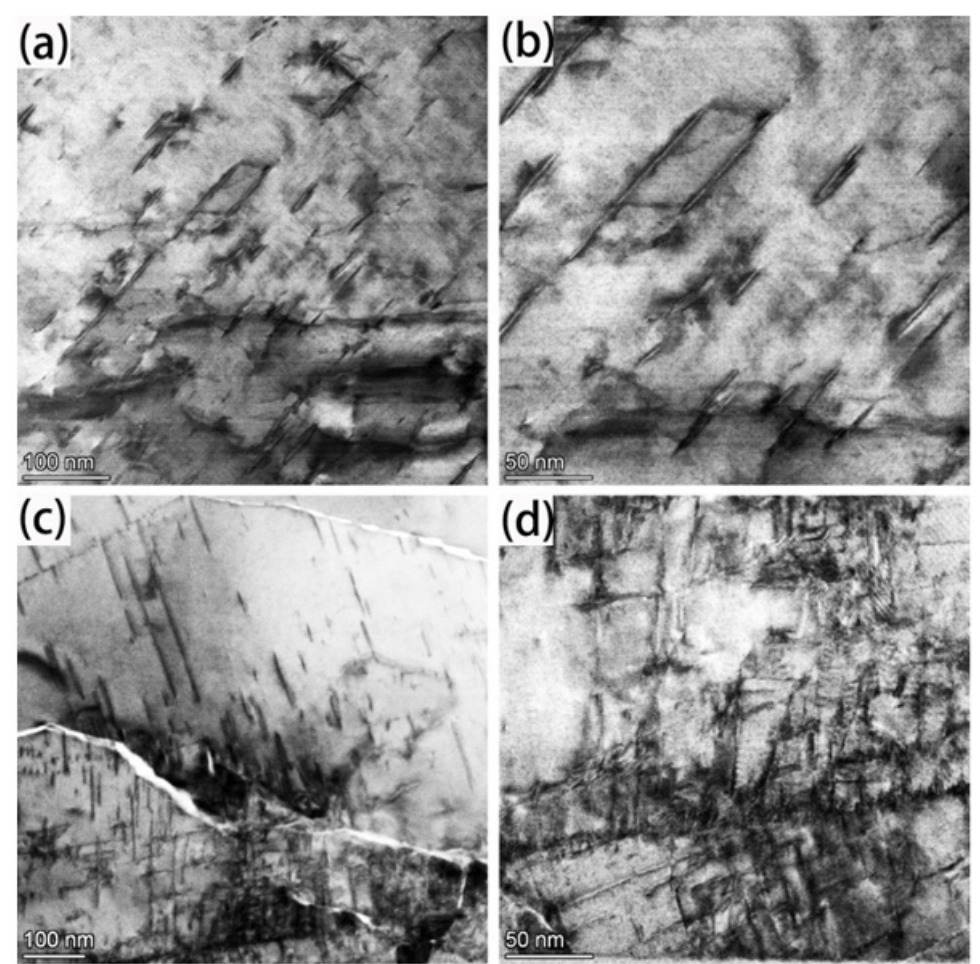

Figure 5. Bright-field TEM microstructure images of the 0.02PSmN-0.68PMN-0.30PT single crystal under $(\mathbf{a}, \mathbf{b})$ alternating current polarization $(\mathrm{ACP})$; (c,d) direct current polarization (DCP).
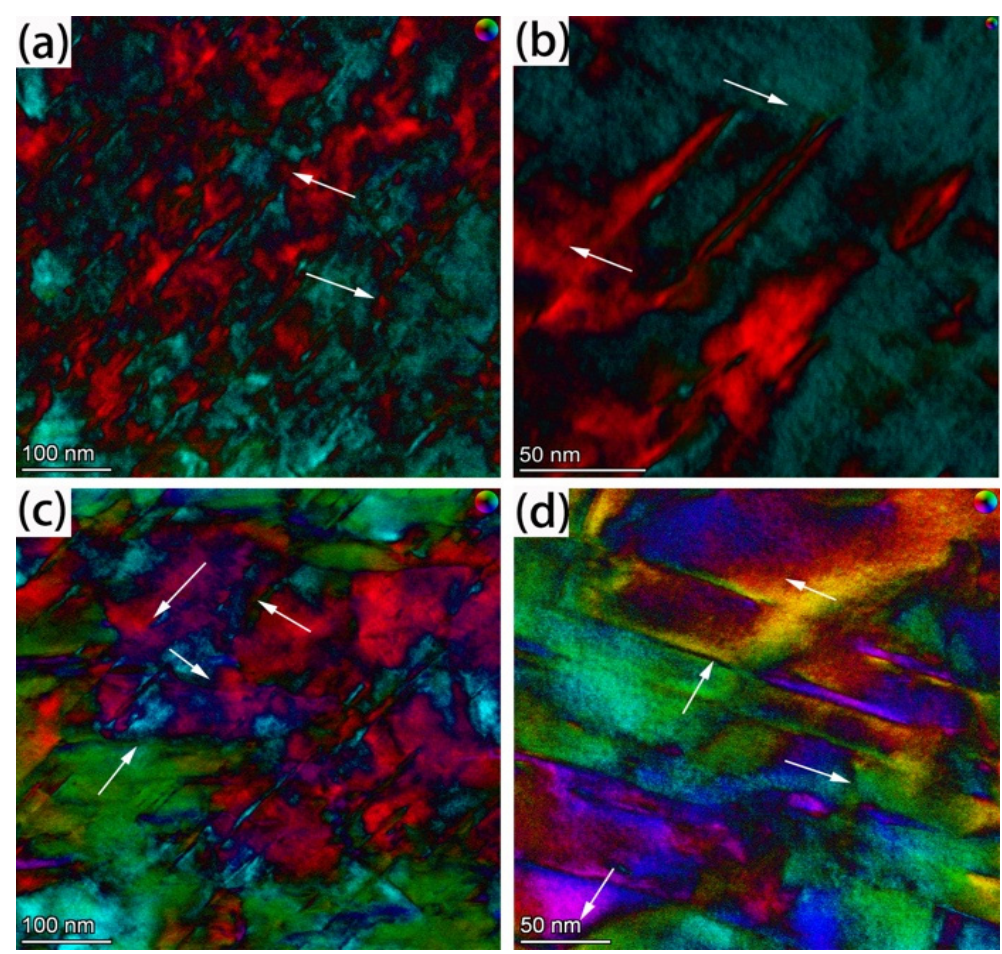

Figure 6. Differential phase contrast (DPC) images of the 0.02PSmN-0.68PMN-0.30PT single crystal under $(\mathbf{a}, \mathbf{b})$ ACP; (c,d) DCP. 


\subsection{Luminescent Property}

A series of polished crystal wafers of 0.5 or $2.0 \mathrm{~mm}$ thickness were fabricated from the crystal boule (b). After the crystal wafers were polarized by DC or AC voltage, the optical transmission spectra of the crystal wafers were measured in the wavelength range of 250 1750 nm (Figure 7). We found that the AC-polarized crystal wafers exhibited a much higher optical transmittance than the DC-polarized samples, especially in the visible light region. The light below a $400 \mathrm{~nm}$ wavelength was completely absorbed because of the optical absorption edge $(\sim 3.10 \mathrm{eV})$, which was similar to most oxygen octahedral perovskites $[27,28]$. The light absorption coefficient of the AC-polarized sample was found to be substantially decreasing at a wavelength longer than $400 \mathrm{~nm}$, whereas the absorption coefficient of the DC-polarized sample remained large and decreased slowly with increasing wavelength. Because of the sharp decreasing of $71^{\circ}$ domain walls [26], the AC-polarized crystal wafers exhibited a desirable optical transmittance even as high as $65 \sim 70 \%$ in the region above $500 \mathrm{~nm}$.

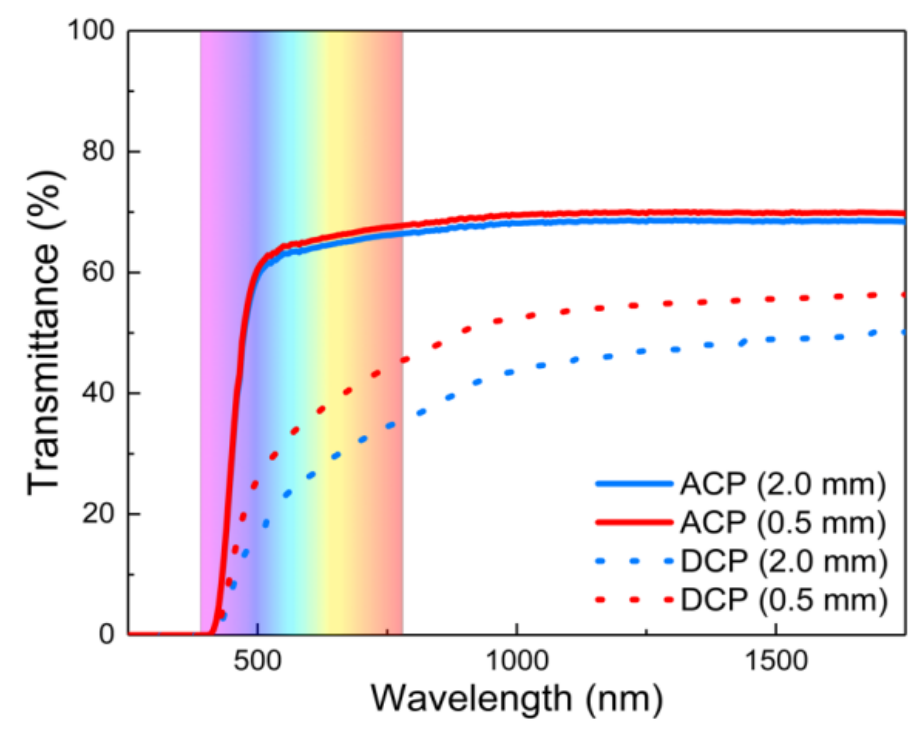

Figure 7. The optical transmittance of the crystal wafers fabricated from the crystal $0.02 \mathrm{PSmN}$ 0.68PMN-0.30PT under ACP and DCP.

The luminescent properties of (a), (b), and (c) crystal wafers were characterized by the emission spectra and excitation spectra shown in Figure 8. Under $408 \mathrm{~nm}$ photonic excitation, the emission spectra in Figure 8a present the four intense emission peaks located at 562, 598, 644, and $705 \mathrm{~nm}$, which were attributed to the doped $\mathrm{Sm}^{3+}$ ions with the characteristic energy level transitions of ${ }^{4} \mathrm{G}_{5 / 2} \rightarrow{ }^{6} \mathrm{H}_{5 / 2},{ }^{4} \mathrm{G}_{5 / 2} \rightarrow{ }^{6} \mathrm{H}_{7 / 2},{ }^{4} \mathrm{G}_{5 / 2} \rightarrow{ }^{6} \mathrm{H}_{9 / 2}$ and ${ }^{4} \mathrm{G}_{5 / 2} \rightarrow{ }^{6} \mathrm{H}_{11 / 2}$, respectively [29]. Using the strongest emission at $598 \mathrm{~nm}$ as the monitor mode, the excitation spectra in Figure $8 \mathrm{~b}$ exhibit a series of spectral lines in the 300 500 nm region, corresponding to the energy level transitions of ${ }^{6} \mathrm{H}_{5 / 2} \rightarrow{ }^{4} \mathrm{D}_{1 / 2}$ $(381 \mathrm{~nm}),{ }^{6} \mathrm{H}_{5 / 2} \rightarrow{ }^{4} \mathrm{~F}_{7 / 2}(408 \mathrm{~nm}),{ }^{6} \mathrm{H}_{5 / 2} \rightarrow\left({ }^{6} \mathrm{P}_{3 / 2},{ }^{4} \mathrm{P}_{5 / 3}\right)(420 \mathrm{~nm}),{ }^{6} \mathrm{H}_{5 / 2} \rightarrow{ }^{4} \mathrm{G}_{9 / 2}(439 \mathrm{~nm})$, ${ }^{6} \mathrm{H}_{5 / 2} \rightarrow{ }^{4} \mathrm{I}_{13 / 2}(468 \mathrm{~nm})$, and ${ }^{6} \mathrm{H}_{5 / 2} \rightarrow{ }^{4} \mathrm{I}_{11 / 2}(480 \mathrm{~nm})$ [26-28]. The emission peaks located at 468 and $480 \mathrm{~nm}$ split into two sub-peaks with weaker peaks at 464 and $474 \mathrm{~nm}$, respectively, which could be attributed to the Stark effect owing to the strong inner electric field of the host crystals [27]. The emission spectra and excitation spectra of (a), (b), and (c) crystal wafers showed an increasing tendency with the luminescent intensity basically with increasing concentrations of the doped $\mathrm{Sm}^{3+}$ ions. 

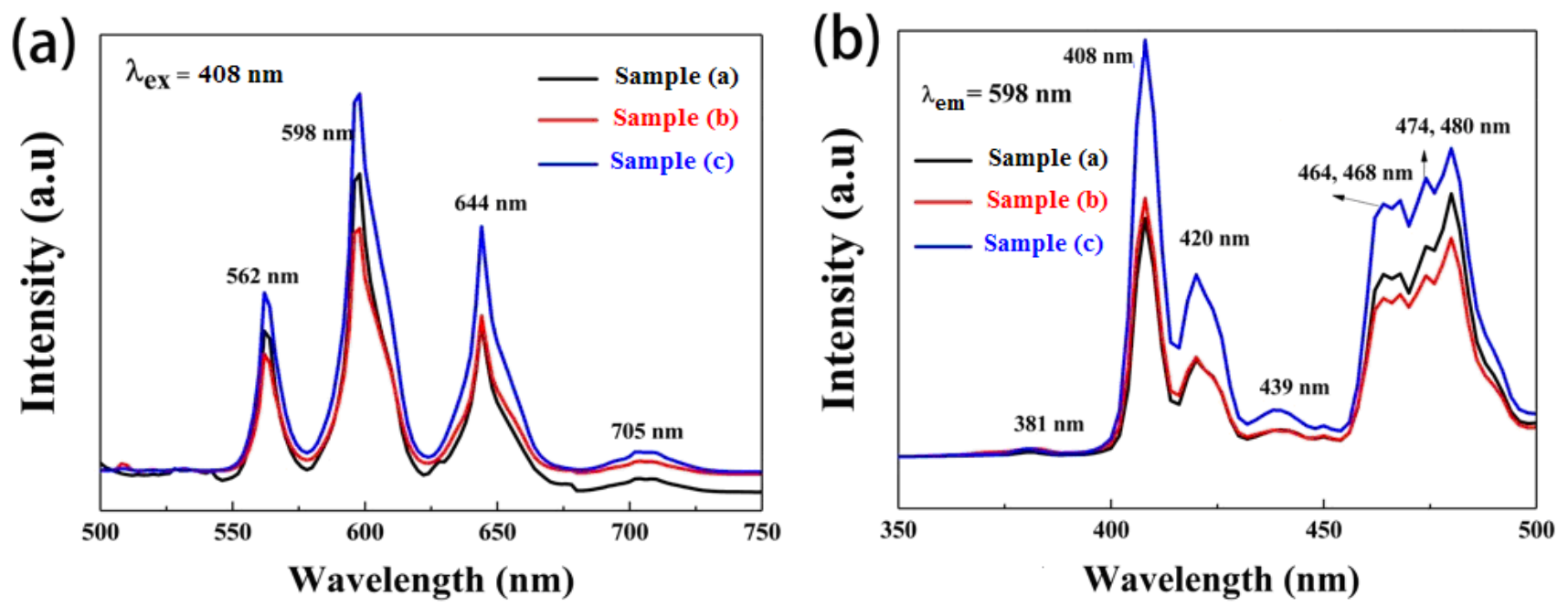

Figure 8. The emission spectra (a) and excitation spectra (b) of the different crystal sample wafers.

\section{Conclusions}

A relaxor-based ferroelectric single crystal with the nominal composition of $x \mathrm{~Pb}\left(\mathrm{Sm}_{0.5} \mathrm{Nb}_{0.5}\right) \mathrm{O}_{3}-(0.7-\mathrm{x}) \mathrm{Pb}\left(\mathrm{Mg}_{1 / 3} \mathrm{Nb}_{1 / 3}\right) \mathrm{O}_{3}-0.3 \mathrm{PbTiO}_{3}(\mathrm{x}=0.01,0.02$ and 0.03$)$ was grown by the vertical Bridgman process. Based on the systematical characterizations of the electrical properties, these novel single crystals with $\mathrm{Sm}^{3+}$ ions doped at $\mathrm{B}$ sites possess a piezoelectric coefficient $d_{33}$ slightly higher in varying degrees than those for the undoped PMN-PT single crystal under the usual direct current polarization. Notably, the crystal wafers had evidently increased $d_{33}$ piezoelectric coefficients for the samples that were subjected to alternating current polarization. However, the ternary single crystals showed a decreased phase transition temperature and Curie temperature compared with the binary single crystal PMN-PT. We found that the crystal wafers polarized with AC voltage also exhibit a desirable optical transmittance, which is associated with the domain structure changes inside the crystal medium. The crystal wafers were verified to show the typical luminescent characteristics due to the effective doped $\mathrm{Sm}^{3+}$ ions in the crystal lattice.

Author Contributions: Conceptualization, F.L., Y.Z. (Yanqing Zheng) and H.C.; methodology, F.L., Y.Z. (Yanqing Zheng) and H.C.; investigation, F.L., Y.Z. (Yan Zhao) and Y.Z. (Yanqing Zheng); resources, F.L., Y.Z. (Yan Zhao) and Z.C.; formal analysis, Y.Z. and (Yan Zhao)Z.C.; validation, F.L.; writing-original draft preparation, F.L.; writing-review and editing, F.L., Y.Z. (Yanqing Zheng) and H.C.; supervision, Y.Z. (Yanqing Zheng) and H.C.; Funding acquisition, Y.Z. (Yanqing Zheng) and H.C. All authors have read and agreed to the published version of the manuscript.

Funding: This work was funded by the National Natural Science Foundation of China (grant No. 21832009), the Prior Project in Key Science \& Technology Program of Zhejiang Province (2009C11144), the Major Innovation Projects on Science and Technology of Ningbo City (2019B10122), and Wong Magna Fund in Ningbo University. The authors are also thankful for the technical cooperation from Shanghai Institute of Ceramics, Chinese Academy of Sciences.

Institutional Review Board Statement: Not applicable.

Informed Consent Statement: Not applicable.

Data Availability Statement: The data presented in this study are available on reasonable request from the corresponding author.

Conflicts of Interest: The authors declare no conflict of interest. 


\section{References}

1. Zhang, S.; Li, F.; Jiang, X.; Kim, J.; Luo, J.; Geng, X. Advantages and challenges of relaxor-PbTiO3 ferroelectric crystals for electroacoustic transducers-A review. Prog. Mater. Sci. 2015, 68, 1-66. [CrossRef]

2. Jaffe, B.; Cook, W.; Jaffe, H. Piezoelectric Ceramics; Academic Press: London, UK, 1971.

3. Haertling, G. Ferroelectric Ceramics: History and Technology. J. Am. Ceram. Soc. 1999, 82, 797-818. [CrossRef]

4. Park, S.E.; Shrout, T.R. Ultrahigh strain and piezoelectric behavior in relaxor based ferroelectric single crystals. J. Appl. Phys. 1997, 82, 1804-1811. [CrossRef]

5. Sun, E.; Cao, W. Relaxor-based ferroelectric single crystals: Growth, domain engineering, characterization and applications. Prog. Mater. Sci. 2014, 65, 124-210. [CrossRef] [PubMed]

6. Service, R.F. Shape-changing crystals get shiftier. Science 1997, 275, 1878. [CrossRef]

7. Chen, J.; Panda, R. Review: Commercialization of piezoelectric single crystals for medical imaging applications. Proc. IUS 2005, 1, 235-240.

8. Zhou, D.; Chen, J.; Luo, L.; Zhao, X.; Luo, H. Optimized orientation of $0.71 \mathrm{~Pb}\left(\mathrm{Mg}_{1 / 3} \mathrm{Nb}_{2 / 3}\right) \mathrm{O}_{3}-0.29 \mathrm{PbTiO} 3$ single crystal for applications in medical ultrasonic arrays. Appl. Phys. Lett. 2008, 93, 073502. [CrossRef]

9. Erturk, A.; Bilgen, O.; Inman, D. Power generation and shunt damping performance of a single crystal lead magnesium niobate-lead zirconate titanate unimorph: Analysis and experiment. Appl. Phys. Lett. 2008, 93, 224102. [CrossRef]

10. Zhang, K.; Choy, S.; Zhao, L.; Luo, H.; Chan, H.; Wang, Y. Shear-mode PMN-PT piezoelectric single crystal resonator for microfluidic applications. Microelectro. Eng. 2011, 88, 1028-1032. [CrossRef]

11. Zhang, S.; Jiang, W.; Meyer, R., Jr.; Li, F.; Luo, J.; Cao, W. Measurements of face shear properties in relaxor-PbTiO $\mathrm{P}_{3}$ single crystals. J. Appl. Phys. 2011, 110, 064106. [CrossRef]

12. Sun, H.; Peng, D.; Wang, X.; Tang, M.; Zhang, Q.; Yao, X. Strong red emission in Pr doped $\left(\mathrm{Bi}_{0.5} \mathrm{Na}_{0.5}\right) \mathrm{TiO}_{3}$ ferroelectric ceramics. J. Appl. Phys. 2011, 110, 016102. [CrossRef]

13. Liu, Y.; He, C.; Yang, X.; Li, X.; Wang, Z.; Huang, Z.; Lai, F.; Long, X. Growth and characterization of $\mathrm{Pb}\left(\mathrm{Lu}_{1 / 2} \mathrm{Nb}_{1 / 2}\right) \mathrm{O}_{3}$ $\mathrm{Pb}\left(\mathrm{Mg}_{1 / 3} \mathrm{Nb}_{2 / 3}\right) \mathrm{O}_{3}-\mathrm{PbTiO}_{3}$ ternary piezo-/ferroelectric crystals. J. Alloys Compd. 2016, 675, 8-14. [CrossRef]

14. He, C.; Li, X.; Wang, Z.; Long, X.; Mao, S.; Ye, Z. Preparation and Characterization of $\mathrm{New} \mathrm{Pb}\left(\mathrm{Yb}_{1 / 2} \mathrm{Nb}_{1 / 2}\right) \mathrm{O}_{3}-\mathrm{Pb}\left(\mathrm{Mg}_{1 / 3} \mathrm{Nb}_{2 / 3}\right) \mathrm{O}_{3}-$ $\mathrm{PbTiO}_{3}$ Ternary Piezo-/Ferroelectric Crystals. Chem. Mater. 2010, 22, 5588-5592. [CrossRef]

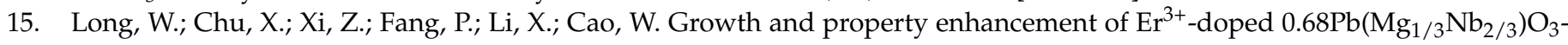
$0.32 \mathrm{PbTiO}_{3}$ single crystal. J. Rare Earths 2018, 36, 832-837. [CrossRef]

16. Yu, H.; Xi, Z.; Fang, P.; Long, W.; Li, X.; Zhang, J.; He, A. Optical properties induced and ferroelectric properties enhanced of the PMN-32PT:Ho relaxor ferroelectric crystals. Optik 2018, 164, 178-185. [CrossRef]

17. Li, F.; Lin, D.; Chen, Z.; Cheng, Z.; Wang, J.; Li, C.; Xu, Z.; Huang, Q.; Liao, X.; Chen, L.Q.; et al. Ultrahigh piezoelectricity in ferroelectric ceramics by design. Nat. Mater. 2018, 17, 349-354. [CrossRef]

18. Li, F.; Cabral, M.J.; Xu, B.; Cheng, Z.; Dickey, E.C.; LeBeau, J.M.; Wang, J.; Luo, J.; Taylor, S.; Hackenberger, W.; et al. Giant piezoelectricity of $\mathrm{Sm}$-doped $\mathrm{Pb}\left(\mathrm{Mg}_{1 / 3} \mathrm{Nb}_{2 / 3}\right) \mathrm{O}_{3}-\mathrm{PbTiO}_{3}$ single crystals. Science 2019, 364, 264-268. [PubMed]

19. Chen, H.; Liang, Z.; Luo, L.; Ke, Y.; Shen, Q.; Xia, Z.; Jiang, C.; Pan, J. Bridgman growth, crystallographic characterization and electrical properties of relaxor-based ferroelectric single crystal PIMNT. J. Alloys Compd. 2012, 518, 63-67. [CrossRef]

20. Jiang, L.; Wang, Z.; Chen, Y.; Chen, P.; Luo, L.; Chen, H. Bright up-conversion emission of Er ${ }^{3+}$-doped lead-free ferroelectric $\mathrm{Na}_{0.5} \mathrm{Bi}_{0.5} \mathrm{TiO}_{3}$ single crystal. Mater. Lett. 2018, 210, 158-160. [CrossRef]

21. Zheng, T.; Luo, L.; Du, P.; Deng, A.; Li, W. Ferroelectric, upconversion emission and optical thermometric properties of colorcontrollable $\mathrm{Er}^{3+}$-doped $\mathrm{Pb}\left(\mathrm{Mg}_{1 / 3} \mathrm{Nb}_{2 / 3}\right) \mathrm{O}_{3}-\mathrm{PbTiO}_{3}-\mathrm{Pb}\left(\mathrm{Yb}_{1 / 2} \mathrm{Nb}_{1 / 2}\right) \mathrm{O}_{3}$ ferroelectrics. J. Eur Ceram. Soc. $2018,38,575-583$. [CrossRef]

22. Yao, Y.; Luo, L.; Zuo, Q.; Li, W.; Zhou, J. The ferroelectric, dielectric and up-conversion photoluminescence properties of ferroelectrics (0.99-x) $\mathrm{PbTiO}_{3}-\mathrm{xPb}\left(\mathrm{Yb}_{1 / 2} \mathrm{Nb}_{1 / 2}\right) \mathrm{O}_{3}-0.01 \mathrm{~Pb}\left(\mathrm{Er}_{1 / 2} \mathrm{Nb}_{1 / 2}\right) \mathrm{O}_{3}$. J. Alloys Compd. 2016, 673, 102-106. [CrossRef]

23. Akca, E.; Duran, C. Fabrication and characterization of $\left(\mathrm{Pb}\left(\mathrm{Mg}_{1 / 3} \mathrm{Nb}_{2 / 3}\right) \mathrm{O}_{3}, \mathrm{~Pb}\left(\mathrm{Yb}_{1 / 2} \mathrm{Nb}_{1 / 2}\right) \mathrm{O}_{3}, \mathrm{PbTiO}_{3}\right)$ ternary system ceramics. Ceram. Int. 2011, 37, 2135-2142. [CrossRef]

24. Zhu, R.; Fang, B.; Zhao, X.; Zhang, S.; Chen, Z.; Ding, J.; Luo, H. Enhancing piezoelectric properties of high-Curie temperature PMN-PH-PT piezoelectric ceramics by citrate method. J. Alloys Compd. 2018, 735, 496-509. [CrossRef]

25. Tian, J.; Han, P.; Payne, D.A. Measurements along the growth direction of PMN-PT crystals: Dielectric, piezoelectric, and elastic properties. IEEE Trans. Ultrason. Ferroelectr. Freq. Control 2007, 54, 1895-1902. [CrossRef]

26. Qiu, C.; Wang, B.; Zhang, N.; Zhang, S.; Liu, J.; Walker, D.; Wang, Y.; Tian, H.; Shrout, T.R.; Xu, Z.; et al. Transparent ferroelectric crystals with ultrahigh piezoelectricity. Nature 2020, 577, 350-354. [CrossRef]

27. Wan, X.; Luo, H.; Wang, J.; Chan, H.; Choy, C.L. Investigation on optical transmission spectra of $(1-\mathrm{x}) \mathrm{Pb}\left(\mathrm{Mg}_{1 / 3} \mathrm{Nb}_{2 / 3}\right) \mathrm{O}_{3}-\mathrm{xPbTiO} 3$ single crystals. Solid State Commun. 2004, 129, 401-405. [CrossRef]

28. Wemple, S. Polarization fluctuations and the optical-absorption edge in $\mathrm{BaTiO}_{3}$. Phys. Rev. B 1970, 2, 2679-2689. [CrossRef]

29. NagarajaNaick, B.; Reddy, P.V.; Damodaraiah, S.; Reddy, A.V.; Ratnakaram, Y.C. Absorption and luminescence studies of Sm3+ ions activated in distinct phosphate glasses for reddish orange light applications. Opt. Mater. 2019, 88, 7-14. [CrossRef] 